\title{
RESPONSABILIDAD PROFESIONAL APLICADA A LA FARMACOVIGILANCIA: UN ESTUDIO DE CASO EN MÉXICO
}

\author{
Germán Novoa-Heckel ${ }^{1}$, Juan Asbún-Bojalil ${ }^{1}$, María de la Luz Sevilla-González ${ }^{1}$
}

Resumen: El sistema internacional de farmacovigilancia recopila información sobre eventos adversos por medicamentos a partir de informes emitidos por los médicos en la práctica clínica. Los reportes de reacciones adversas a medicamentos (RAM) son escasos. Diseńamos un cuestionario basado en una escala de Likert de cinco puntos. El cuestionario, de 24 preguntas, se administró a una muestra de médicos. Las respuestas de los cuestionarios indican que es importante contar con sistemas de farmacovigilancia. A pesar de ello, casi tres cuartos de los encuestados no sabían cómo informar de una reacción adversa y dos quintos no deseaban hacerlo. Tres cuartas partes de los encuestados expresaron que la instrucción sobre la elaboración de informes debería comenzar durante la carrera de Medicina. Los resultados sugieren que la elaboración de informes de RAM debe incluirse en la educación médica. Es necesario establecer protocolos que aseguren que los lineamientos para informar RAM sean fáciles de seguir. Es recomendable que los programas de capacitación y educación médica continua generen una conciencia de responsabilidad frente al reporte de RAM.

Palabras clave: farmacovigilancia, sistemas de reporte de reacciones adversas a medicamentos, encuesta, cuestionario, responsabilidad

Professional responsibility applied to drug surveillance: a study case in México

\begin{abstract}
The international pharmacovigilance system collects adverse drug events reported by physicians in their clinical practice. Adverse drug reactions (ADR) reported are scarce. We designed a questionnaire based on a 5-point Likert scale. The questionnaire (24 questions) was administered to a sample of physicians in Mexico City. Questionnaire responses indicated that having pharmacovigilance systems is important. Despite this, nearly three quarters of respondents did not know how to report an adverse drug reaction, and two-fifths were not willing to do so. Three quarters believed that education regarding reporting and its methods should be initiated during medical school. The results from our questionnaire suggest that medical school training in ADR report writing should be implemented. Good protocols have to be in place to ensure that ADR report forms have easy-to-follow guidelines and are easy to complete. It is recommended that the programs of training and medical education generate conscience of responsability towards the report of ADRs.
\end{abstract}

Key words: pharmacovigilance, adverse drug reaction reporting systems, survey, questionnaire, responsibility

Responsabilidade profissional aplicada à farmacovigilância: um estudo de caso no México

Resumo: O sistema internacional de farmacovigilância recopila informação sobre eventos adversos por medicamentos a partir de informes emitidos pelos médicos na prática clínica. Os informes de reaçôes adversas a medicamentos (RAM) são escassos. Idealizamos um questionário baseado numa escala de Likert de cinco pontos. O questionário, de 24 perguntas, foi administrado a uma mostra de médicos. As respostas dos questionários indicam que é importante contar com sistemas de farmacovigilância. Apesar disso, quase três quartos dos entrevistados não sabiam como informar uma reaçáo adversa e dois quintos não desejavam fazê-lo. Três quartas partes dos entrevistados expressaram que a instrução sobre a elaboração de informes deveria começar durante o curso de Medicina. Os resultados sugerem que a elaboração de informes de RAM deve ser incluido na educação médica. É necessário estabelecer protocolos que assegurem que as orientaçóes para informar RAM sejam fáceis de seguir. É recomendável que os programas de capacitação e educação médica contínua gerem uma consciência de responsabilidade frente ao informe de RAM.

Palavras-chave: farmacovigilância, sistemas de informe de reações adversas a medicamentos, sondagem, questionário, responsabilidade

\footnotetext{
${ }^{1}$ División de Posgrado e Investigación, Escuela Superior de Medicina, Instituto Politécnico Nacional, México Correspondencia: luzmariompi@gmail.com
} 


\section{Introducción}

El presente documento pondera la responsabilidad profesional como punto de partida clave para incidir el aumento de reacciones adversas a los medicamentos (RAM) en los países de América Latina. Para abordar el tema de manera más sucinta, hemos dividido el artículo en dos partes: En la primera se plantea brevemente cómo surge en el mundo la iniciativa de la farmacovigilancia y cómo se relaciona con el principio de responsabilidad desde la propuesta de Hans Jonas, la cual guía al lector hacia una reflexión sobre la necesidad de entender que se "ha abierto una dimensión totalmente nueva de relevancia ética no prevista en las perspectivas y cánones de la ética tradicional" (1). La segunda parte del documento muestra resultados de una investigación realizada en México D.F., donde el objetivo principal fue indagar acerca de los conocimientos y actitudes respecto del tema de la farmacovigilancia prevalentes en 426 médicos entrevistados.

En todo el mundo, la farmacovigilancia es una iniciativa global emprendida hace más de 45 años, con la finalidad de detectar oportunamente los riesgos inherentes al uso de fármacos y tecnologías médicas. La sede internacional se localiza en Uppsala, Suecia(2,3), a donde se envían los reportes emitidos en cada país. Un reporte de reacciones adversas a medicamentos, emitido por un clínico (y enviado primero a las autoridades locales), se denomina "reporte espontáneo". Esta es la manera más apropiada de producir las llamadas señales que guían la detección de problemas de seguridad en el uso de algún fármaco. México ha formado parte de este programa internacional desde hace alrededor de 15 años. La Norma Oficial Mexicana NOM-220-SSA1-2002, "Instalación y operación de la farmacovigilancia"(4), establece que los médicos clínicos están obligados a informar eventos adversos por uso de medicamentos. Las modificaciones hechas a la legislación en 2013 establecen que los ciudadanos deberían informar eventos adversos por medicamentos, pero solo los profesionales de la salud están obligados a hacerlo.

La baja tasa de reportes es una gran limitante para el sistema; se estima que, internacionalmente, solo 6\% de las RAM se informan. Diversos es- tudios publicados globalmente por investigadores que participan en el programa (generadores de reportes) han investigado las limitaciones de este tipo de informes y las causas de dichas limitaciones, con la finalidad de incrementar su producción en el ámbito nacional(5,6-11).

En México se ha estructurado una participación tripartita entre gobierno, instituciones de salud y profesionales de la salud. La COFEPRIS, como institución de gobierno, junto con los institutos de salud y hospitales, han generado en el país una estructura vinculante con miras a mejorar el reporte de RAM. A pesar de que existen programas de capacitación en farmacovigilancia, aún son incipientes y ha quedado pendiente trabajar sobre una sensibilización estructural para responsabilizar a los médicos en cuanto a las RAM y generar una cultura del informe a partir de la educación médica continua o la capacitación permanente, en la que se fomente de forma permanente una ética basada en el principio de responsabilidad. Hans Jonas hace una reflexión sobre la visión antropocentrista en el pasado, en la que los hombres tenían una directriz única, al someter la naturaleza al beneficio del ser humano. Si partimos de una visión bioecocentrista, entenderemos que el planeta es uno solo y el avance de la ciencia y la tecnología no necesariamente van aparejados a un beneficio humanista. Partiendo de este supuesto, ¿cuál es el papel del médico y hasta dónde llega su responsabilidad al "diagnosticar" un medicamento? Es innegable que el desarrollo de la industria farmacéutica ha alargado la sobrevida del ser humano, pero también es necesario apuntar que el acceso a los medicamentos, por su costo, deja fuera a un número importante de pacientes. El médico necesita comprender la importancia del reporte de RAM y así cumplir con su responsabilidad profesional en aras de un beneficio común entre el avance científico, el desarrollo profesional y el bienestar de los pacientes

\section{Intervención en México}

México no es la excepción a la regla de los informes escasos. A pesar de una tendencia creciente al paso de los años, el número de reportes espontáneos de RAM recibidos por el Sistema de Farmacovigilancia sigue siendo insuficiente(9) (en 2009, el año de mayor índice de reportes en 
México, el promedio fue de aproximadamente uno por año por cada diez médicos). Este fenómeno constituye el tema de nuestra investigación.

López-González et al.(5) afirman que las RAM son un problema de salud pública en cuanto a mortalidad, morbilidad y costos. Parten del modelo teórico conocido como "los siete pecados capitales" (6-8) para explicar las razones principales para no reportar y determinar la manera en que el conocimiento y las actitudes de los médicos influyen en la generación de informes. Se han distribuido cuestionarios autoadministrados entre los médicos para ayudar a definir el problema y sugerir soluciones(12).

Nuestro objetivo inicial de trabajo fue desarrollar un cuestionario autoadministrado, basado en otros cuestionarios existentes(12-15), con el fin de investigar conocimientos y actitudes respecto del tema de la farmacovigilancia prevalentes entre los médicos en México. El cuestionario se produjo originalmente en español, pero también está disponible en inglés.

\section{Métodos}

\section{Sujetos de estudio}

Médicos (generales y especialistas) de dos hospitales elegidos al azar participaron tanto en la validación inicial como en el sondeo posterior. Todos ellos habían ejercido como clínicos en la Ciudad de México durante más de un año. Distribuimos los cuestionarios al personal en distintos momentos de las sesiones matutinas llevadas a cabo en los auditorios de los hospitales. El cuestionario se aplicó a un número creciente de médicos (30, 124 y 250) a lo largo de un proceso de validación de ocho meses de duración. El cuestionario final lo respondieron 558 médicos (incluyendo 124 encuestas piloto).

\section{Consideraciones éticas y legales}

El protocolo de estudio se presentó previamente ante el comité de ética de la Escuela Superior de Medicina del Instituto Politécnico Nacional. El mismo comité aprobó el presente estudio. El comité no consideró necesario un consentimiento informado. Todos los datos fueron encriptados y mantenidos por los investigadores, siguiendo las pautas de las buenas prácticas en documentación, para garantizar confidencialidad total.

El cuestionario autoadministrado tomó en cuenta los lineamientos de farmacovigilancia establecidos para los médicos mexicanos por la Secretaría de Salud, a través de la Comisión Federal para la Protección contra Riesgos Sanitarios (COFEPRIS) en 2004 (NOM-220-SSA1-2002 \$5)(4).

\section{Desarrollo del cuestionario}

Tres expertos en farmacovigilancia generaron el primer banco de preguntas, orientado hacia la determinación del conocimiento sobre el sistema nacional de farmacovigilancia y las actitudes de los médicos hacia este. El cuestionario fue formulado y se adaptó a una escala de Likert(12-15). Se exploraron tres temas: conocimiento e importancia del sistema, razones para no reportar (y conocimiento), y posibles soluciones y razones para reportar (y conocimiento). Se asignaron valores numéricos entre 1 y 5 para cada respuesta: 5 correspondía a "totalmente de acuerdo", 4 a "de acuerdo", 3 para "ni de acuerdo ni en desacuerdo", 2 correspondía a "en desacuerdo", y 1 a "totalmente en desacuerdo". El máximo puntaje posible para el cuestionario piloto de 30 preguntas fue de 150 (puntuación máxima a obtener por pregunta $=5$ puntos). En el cuestionario final de 24 preguntas, el máximo puntaje posible fue de 120 puntos. De esta manera se pudo calcular un índice de respuestas total para cada encuestado. Una conversión de este índice dio como resultado el porcentaje final de respuestas "correctas" en relación con un $100 \%$ correspondiente al valor máximo e ideal. La formulación de las preguntas en sentido positivo o negativo se ajustó para lograr el puntaje máximo mencionado. Para asegurar que el valor óptimo predeterminado de 120 se alcanzara cuando todas las respuestas fueran "correctas", se invirtió el sentido de la redacción de las preguntas respondidas con "totalmente de acuerdo" (para el análisis del puntaje) cuando la respuesta óptima según los autores fuera "totalmente en desacuerdo". En los casos en que la respuesta ideal era "totalmente en desacuerdo", se asignaron valores de 5 a 1 a las respuestas. La formulación inversa se aplicó a las siguientes preguntas seleccionadas al azar: 1, 2, 5, 14, 15, 20, 
$21,22,24,28,30$ (preguntas $1,11,12,16,17$, 19, 23, en el cuestionario final de 24 preguntas). La inversión se realizó para evitar posibles sesgos.

\section{Análisis estadistico}

1. Resultados de la validación. Los datos obtenidos de los cuestionarios se ingresaron a una base de datos SPSS (versión 13.0, SPSS, Inc., Chicago, IL, EUA) (16). Se tabularon y clasificaron las respuestas para medir fiabilidad y consistencia interna. Se usó el alfa de Cronbach $(17,18)$ y la prueba t de Student pareada para validación y comparación prueba/posprueba. Se evaluó la concordancia comparando las respuestas 9 y 19, que fueron específicamente diseñadas como repeticiones.

Para el análisis del cuestionario, las preguntas se clasificaron de acuerdo a cada una de las dimensiones que menciona Inman(7). Luego se les asignó el máximo puntaje posible ("totalmente de acuerdo") en la escala Likert de 5 puntos(13). Estos valores se tabularon contra el máximo valor posible (valor óptimo $=150$ puntos).

2. Determinación de la fiabilidad y consistencia del cuestionario. En la aplicación piloto, se entregó el cuestionario a 124 médicos generales y especialistas. La muestra incluyó entre 100 y 200 sujetos, en concordancia con los requerimientos teóricos(12).

Se aplicó la prueba de consistencia interna (concordancia; valor obtenido: 1.097), para estimar la fiabilidad en términos del grado en que diferentes secciones del cuestionario evaluaban los mismos atributos o dimensiones.

El número de respuestas "correctas" y de errores (preguntas sin contestar y su frecuencia) se usaron para analizar la fiabilidad y sensibilidad del cuestionario en términos porcentuales. Las respuestas de cada encuestado se conjugaron en un porcentaje global, para después analizar e interpretar la congruencia de los resultados.

\section{Aplicación del cuestionario final}

El cuestionario final validado de 24 preguntas fue respondido por 558 médicos. Las preguntas relacionadas con los factores de Inman para no reportar(6) se compararon con datos internacio- nales sobre los mismos factores.

Las 24 preguntas utilizadas en el cuestionario.

1. Hace falta mayor compromiso/obligación de los médicos para reportar las reacciones adversas. Totalmente de acuerdo/De acuerdo / $\mathrm{Ni}$ de acuerdo ni en desacuerdo/En desacuerdo /Totalmente en desacuerdo

2. El sistema de reporte de Farmacovigilancia no reviste importancia ni trascendencia para mi trabajo.

3. El reporte de Farmacovigilancia no reviste importancia ni trascendencia para el país.

4. Hay muchos asuntos más importantes dentro de la práctica médica por atenderse.

5. El reporte de Farmacovigilancia le atañe más a las autoridades que a nosotros los médicos.

6. No encuentro una buena razón para hacer el reporte de forma rutinaria.

7. El reporte hace que se exponga mi práctica clínica de manera innecesaria.

8. Realmente no sé cómo realizar un reporte de reacciones adversas espontáneas.

9. Solamente aquellas reacciones adversas medicamentosas que demuestran una relación causa-efecto deben ser reportadas.

10. Las reacciones adversas de un medicamento ya son conocidas cuando el medicamento llega al mercado, puesto que solamente se comercializan medicamentos seguros

11. Los médicos debiéramos contribuir al avance general del conocimiento médico a través del reporte de reacciones adversas medicamentosas

12. En realidad es muy difícil el determinar si un medicamento es el responsable de una reacción adversa.

13. El reporte de reacciones adversas medicamentosas requiere de mucho tiempo y atención. 
14. No sé dónde encontrar el formato de Reporte de Reacciones Adversas a Medicamento

15. El reporte es burocrático y complicado.

16. Al reporte lo considero valioso en algunos casos de controversia legal.

17. El formato de reporte debiera ser más amigable en su diseño.

18. Solamente un sistema de castigo-recompensa hará que los médicos reporten las reacciones adversas a los medicamentos.

19. Con el reporte se puede contribuir al conocimiento médico.

20. Solamente cuando hay una relación evidente entre el medicamento y la reacción se debe reportar.

21. Es la severidad de la reacción la que decide si debemos o no reportar la reacción adversa medicamentosa.

22. Solamente deben reportarse reacciones adversas previamente desconocidas.

23. Tiene que educarse lo relacionado con el reporte en la facultad para que funcione.

24. Realmente no sé cuál es el alcance del reporte ante las autoridades.

Los datos se vaciaron en una base de datos de Excel (Microsoft, Inc., Redmond, WA, EUA) y después se transfirieron al programa estadístico SPSS, versión 13.0 (SSPS, Inc., Chicago, IL, EUA) para realizar los análisis correspondientes. Para los datos demográficos se aplicaron parámetros estadísticos simples (promedio, media, mínimos y máximos, porcentajes). Se empleó la prueba de $\mathrm{CH}^{2}$ para medir en nivel asociación de los encuestados entre sí. Las puntuaciones finales del sondeo se compararon usando pruebas $t$ de Student con distribución de dos colas. Se consideró estadísticamente significativo un valor de $p$ $<0.05$.

\section{Resultados}

La tabla 1 muestra las características demográficas de los encuestados.

Tabla 1. Características demográficas de los encuestados.

\begin{tabular}{|c|c|c|c|}
\hline & Valor & Mín./máx. & D.E. \\
\hline $\mathrm{N}$ & 426 & & \\
\hline Hombres/mujeres & $180 / 246$ & $21 / 64$ & 9.72 \\
\hline $\begin{array}{c}\text { Edad media } \\
\text { (ańos) }\end{array}$ & 31.42 & $1 / 35$ & 9.10 \\
\hline $\begin{array}{c}\text { Experiencia } \\
\text { media (años) }\end{array}$ & 7.71 & $3 / 30$ & 8.33 \\
\hline $\begin{array}{c}\text { Número de } \\
\text { pacientes/día } \\
\text { (media) }\end{array}$ & 15.70 & & \\
\hline
\end{tabular}

Se recolectó un total de 558 cuestionarios. Tras eliminar aquellos con información incompleta, quedaron 426 completos para incluir en el análisis estadístico final. Así, el índice de respuesta final para el sondeo fue de 426/558=76,3\%.

El gráfico 1 muestra las respuestas agrupadas en tres categorías, con el fin de facilitar la interpretación. Las respuestas "totalmente de acuerdo" y "de acuerdo" se agrupan en una sola categoría; "totalmente en desacuerdo" y "en desacuerdo" se agrupan en otra. Las respuestas "ni de acuerdo ni en desacuerdo" se reportan por separado como "indeciso".

Gráfico 1.

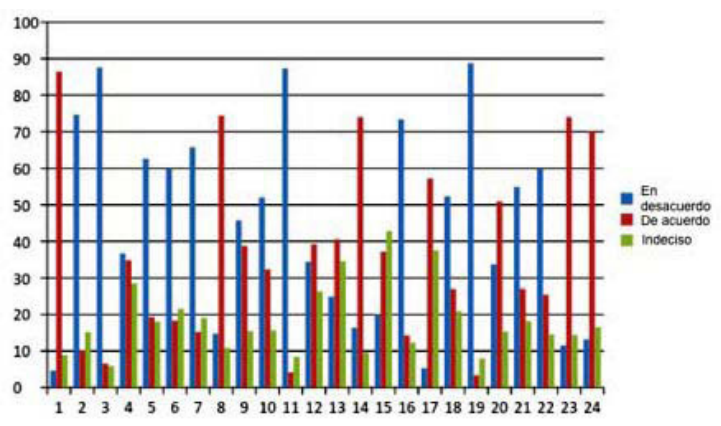


Preguntas 1-8 (tabla 2): El cuestionamiento principal aquí tiene que ver con la importancia del programa, qué tanto la comprenden los médicos, y el conocimiento de los médicos sobre el programa. La mayoría de los encuestados están de acuerdo en que reportar RAM es importante (pregunta 2). Sin embargo, casi el $75 \%$ no saben cómo hacer un reporte. Las respuestas al enunciado de la pregunta 6, "No encuentro una buena razón para hacer el reporte de forma rutinaria", muestran que dos quintas partes de los encuestados no están del todo dispuestos a reportar.

Tabla 2. Factores (favorables y adversos) evaluados en el sondeo: conocimiento e importancia del sistema.

Preguntas 9-16 (tabla 3): El segundo grupo de preguntas se refiere a las razones para no informar, tema central del estudio. Aproximadamente la mitad de los encuestados cree que solamente se deben reportar las reacciones adversas que demuestran una relación causa-efecto (pregunta 9), y que las RAM ya son conocidas cuando el medicamento llega al mercado (pregunta 10), lo que revela ignorancia. En la pregunta 15, el 37\% de los encuestados respondió que hacer reportes es burocrático y complicado; más del $42 \%$ no sabía, y solo $20 \%$ no lo consideraban burocrático, lo que muestra una posible tendencia a compensar mediante pretextos. Se observa una tendencia similar en la pregunta 13, en la que se afirma que los reportes requieren mucho tiempo y atención.

Tabla 3. Factores (favorables y adversos) evaluados en el sondeo: razones para no reportar.

Preguntas 17-24 (tabla 4): El último grupo de preguntas está orientado a buscar razones para reportar y posibles soluciones a la falta de reportes. La pregunta 18 investiga si un sistema de premios y castigos estimularía la generación de reportes. Más de la mitad (52,30\%) estuvo en desacuerdo. La afirmación de que el informe debería incluirse como parte de la educación médica para que el sistema funcionara (pregunta 23) fue suscrita por el 74\% de los encuestados. Las preguntas 19-22 abordaron de nuevo algunos pretextos conocidos que respaldan las creencias que obstruyen los reportes; a su vez, estas pueden erradicarse mediante intervenciones educativas.
Tabla 4. Factores (favorables y adversos) evaluados en el sondeo: posibles soluciones y razones para reportar.

La tabla 5 agrupa las respuestas según los factores para no reportar de Inman, esto con el fin de determinar su relevancia con mayor precisión. La importancia de los factores para no informar de Inman (6-8) se indica por su ponderación relativa (en \%).

Tabla 5. Factores que intervienen en la falta de reportes (conocimiento y actitudes, favorables y adversos) según lo indagado en el cuestionario.

(Nota: no se indagó sobre culpa, ambición de publicar, incentivos económicos y timidez, ya que estos factores no se consideraron relevantes en México al momento del estudio).

\section{Discusión}

A partir del primer grupo de preguntas (1-8), acerca del conocimiento del sistema y su importancia, queda claro que, mientras que los informes son un tema importante en México y en todo el mundo, es necesario incrementar el compromiso individual. El grado de conocimiento que se alcanza acerca del sistema no significa que haya un cambio de práctica. Se tiene que fomentar la conciencia de responsabilidad médica y las ventajas que se obtendrán del informe en el profesional y en los pacientes. Las actitudes tienen entonces que cambiar, y deben adicionalmente encauzarse a un bien común. Las opiniones están divididas cuando se trata de la prioridad de los reportes, aunque se considera importante. No se cree que correspondan exclusivamente a las autoridades. Parece que las razones para incorporar los reportes a la práctica rutinaria son insuficientes.

La razón principal para no reportar (preguntas 9-16) radica en el desconocimiento del proceso por parte de los médicos. Muchos creen que únicamente se informan los RAM que muestran una relación causa-efecto evidente, y que solo se comercializan los medicamentos seguros. La gran mayoría está de acuerdo en que es importante instruir a los médicos sobre los casos que deben reportarse. Muchos expresaron que completar el informe toma demasiado tiempo o estaban in- 
decisos al respecto. Una gran mayoría no sabía dónde encontrar el formato de informe; esto no debería ser difícil de modificar, al igual que otros factores relacionados con el conocimiento. Este aspecto señala la falta de generación de programas intensivos que fortalezcan la capacidad de conocer el proceso de forma más amigable.

Hubo opiniones divididas en cuanto a si informar es un proceso burocrático. Algunos lo perciben así, mientras que otros están indecisos, y solo unos pocos consideran que no lo es. En general, muchos médicos no reportan o desconocen el sistema debido a una falta de experiencia con los informes.

Respecto del tercer grupo de preguntas, sobre posibles soluciones y razones para reportar (preguntas 17-24), encontramos que los informes son valiosos para casi tres cuartas partes de los encuestados. A la mayoría le gustaría que el formato fuera más amigable (pero muchos no estaban seguros). La mayoría (poco más del 50\%) no considera que un sistema de premios y castigos aumentaría su frecuencia. Según la gran mayoría, los reportes contribuyen al avance del conocimiento médico. Más de la mitad de los encuestados creen que es necesario que exista una relación entre el evento adverso y el medicamento para que se emita un reporte. Además, casi el 50\% cree que es la severidad de la reacción lo que determina la necesidad de informar. Estas creencias son erróneas y es evidente que deben ser atendidas.

También observamos desconocimiento respecto del enunciado "solamente deben reportarse reacciones adversas previamente desconocidas". Casi tres cuartas partes de los encuestados están de acuerdo en que el tema de los informes debería ser abordado en la carrera de Medicina. Un factor clave en la falta de motivación es el desconocimiento de su importancia.

Las creencias erróneas que revela nuestro sondeo coinciden con las que se informan en estudios anteriores (19,6). Los investigadores han dividido las razones para no reportar en intrínsecas y extrínsecas(10). La primera clase se refiere a los conocimientos y actitudes relacionados con la educación médica que "condicionan la práctica de reportar"; la segunda, a "factores asociados a la interacción de los profesionales en su ambiente de trabajo", en particular, interacciones con la industria médica, la administración de la salud y los pacientes, quienes "también serán importantes en las condiciones para reportar". Esto se basa en el modelo "conocimientos-actitudes-prácticas", propuesto por Islam(20) y Hong(21).

El fenómeno de la falta de informes, que ocurre en mayor o menor medida en todos los países del mundo, ha sido estudiado desde hace muchos años $(5,22)$. Aagaard et al.(23) informan recientemente que el índice de reportes es mayor en los países con ingresos altos: 130 reportes por millón de habitantes; en países de ingresos medio-altos, 17; en países de ingresos medio-bajos, 12, y 3 reportes en países de bajos ingresos. Se observa un amplio rango de variación al interior de cada grupo de países (por ejemplo, de 261 a 9 informes por millón de habitantes en los países de ingreso medio-alto, en 2008). La estructura organizacional y los recursos financieros de cada centro nacional de farmacovigilancia también desempeñan un papel importante.

Hasta donde sabemos, ningún cuestionario de este tipo había sido aplicado antes en México. El sondeo no es representativo de todos los médicos; es deseable que se incluya más médicos procedentes de contextos más diversos, como hospitales y consultorios privados. También es necesario incluir otras ciudades y Estados del país para que el estudio sea significativo en términos estadísticos.

Concluimos que los médicos mexicanos encuestados tienen un conocimiento insuficiente del sistema de reportes de RAM. Los principales aspectos que deben abordarse para estimular la generación de estos informes incluyen problemas técnicos (conocimientos y habilidades) y actitudes de los médicos (en menor grado). Creemos que tanto su cantidad como su calidad de los reportes puede mejorarse a través de la educación durante la carrera de Medicina. Es deseable que Centro Nacional de Farmacovigilancia contribuya a estas acciones, enfatizando sus ventajas. Sabemos que los médicos pertenecientes al sistema de salud nacional están obligados a llenarlos a diario. Resulta necesario implementar protocolos que aseguren que los lineamientos para los reportes sean fáciles 
de seguir y que sea sencillo completar los formatos. Los presentes resultados deberán ser corroborados en investigaciones ulteriores.

Es necesario crear programas de capacitación para incrementar la tasa de reporte. Si bien México ha generado estrategias nacionales por medio de la COFEPRIS, se requiere generar una conciencia de responsabilidad profesional e institucional para ir creando en generaciones venideras una cultura del reporte.

Si la razón principal para no reportar radicó en el desconocimiento del proceso, entonces se requiere capacitar y sensibilizar más al médico sobre su importancia en los niveles individual, hospitalario o institucional.

En el proceso de globalización los reportes son un tema importante. Es necesario incrementar el compromiso en los tres niveles, individual, institucional y nacional, con el fin de generar una cultura del reporte y sus beneficios, estas acciones harán que, a corto o mediano plazo, el objetivo del aumento del número de reportes en países de América latina beneficien a todos por igual.
El estudio concluye que los médicos mexicanos tienen un conocimiento insuficiente del sistema de informes de RAM. Es necesario crear programas de capacitación para incrementar la tasa de reporte. Si bien México ha generado estrategias nacionales por medio de la COFEPRIS, se requiere generar una conciencia de responsabilidad profesional e institucional para ir creando en generaciones venideras una cultura del reporte.

\section{Agradecimientos}

El presente trabajo contó con el apoyo de una beca concedida al Dr. Novoa-Heckel por parte de CONACYT México. El estudio se llevó a cabo a través del programa de Doctorado en Investigación en Medicina (DIM), Sección de Estudios de Posgrado e Investigación (SEPI), de la Escuela Superior de Medicina (ESM) del Instituto Politécnico Nacional (IPN). El trabajo de los autores es independiente del financiamiento. Agradecemos la cooperación de todos los participantes que hicieron posible este estudio.

Tabla 1. Características demográficas de los encuestados.

\begin{tabular}{|l|l|l|l|}
\hline & Valor & Mín./máx. & D.E. \\
\hline $\mathrm{N}$ & 426 & & \\
\hline Hombres/mujeres & $180 / 246$ & & \\
\hline Edad media (años) & 31.42 & $21 / 64$ & 9.72 \\
\hline Experiencia media (años) & 7.71 & $1 / 35$ & 9.10 \\
\hline Número de pacientes/día (media) & 15.70 & $3 / 30$ & 8.33 \\
\hline
\end{tabular}

Tabla 2. Factores (favorables y adversos) evaluados en el sondeo: conocimiento e importancia del sistema.

\begin{tabular}{|l|l|l|l|l|l|}
\hline $\begin{array}{l}\text { Factor para reportar / no } \\
\text { reportar }\end{array}$ & Pregunta & $\begin{array}{l}\text { Favorable } \\
(\%) \text { en } \\
\text { desacuerdo }\end{array}$ & $\begin{array}{l}\text { Adverso } \\
(\%) \text { de } \\
\text { acuerdo }\end{array}$ & $\begin{array}{l}\text { Indeciso/ } \\
\text { no sabe } \\
(\%)\end{array}$ & Comentarios \\
\hline $\begin{array}{l}\text { Los médicos están obligados a } \\
\text { reportar reacciones adversas a } \\
\text { medicamentos. }\end{array}$ & 1 & 4.71 & 86.41 & 8.88 & $\begin{array}{l}\text { El 86\% está de acuerdo } \\
\text { en que se necesita mayor } \\
\text { compromiso y obligación de } \\
\text { reportar. }\end{array}$ \\
\hline $\begin{array}{l}\text { El sistema de reporte de } \\
\text { farmacovigilancia no reviste } \\
\text { importancia ni trascendencia } \\
\text { para mi trabajo. }\end{array}$ & 2 & 74.68 & 10.13 & 15.19 & $\begin{array}{l}\text { Cerca del 75\% está de } \\
\text { acuerdo en la importancia. } \\
\text { El resto están indecisos o la } \\
\text { niegan. }\end{array}$ \\
\hline
\end{tabular}




\begin{tabular}{|l|l|l|l|l|l|}
\hline $\begin{array}{l}\text { El reporte de } \\
\text { farmacovigilancia no reviste } \\
\text { importancia ni trascendencia } \\
\text { para el país. }\end{array}$ & 3 & 87.55 & 6.50 & 5.96 & $\begin{array}{l}\text { Mayormente de acuerdo } \\
\text { en la importancia de un } \\
\text { porcentaje alto de reportes. }\end{array}$ \\
\hline $\begin{array}{l}\text { Hay muchos asuntos más } \\
\text { importantes dentro de la } \\
\text { práctica médica por atenderse. }\end{array}$ & 4 & 36.68 & 34.85 & 28.47 & $\begin{array}{l}\text { Parece no haber consenso } \\
\text { sobre la importancia } \\
\text { en relación con otras } \\
\text { prioridades: } 1 / 3 \text { de acuerdo, } \\
1 / 3 \text { en desacuerdo. }\end{array}$ \\
\hline $\begin{array}{l}\text { El reporte de } \\
\begin{array}{l}\text { Farmacovigilancia le atańe } \\
\text { más a las autoridades que a } \\
\text { nosotros los médicos. }\end{array}\end{array}$ & 5 & 62.64 & 19.31 & 18.05 & $\begin{array}{l}\text { Casi dos tercios están de } \\
\text { acuerdo en que no es un } \\
\text { asunto que atańa sólo a las } \\
\text { autoridades. }\end{array}$ \\
\hline $\begin{array}{l}\text { No encuentro una buena } \\
\text { razón para hacer el reporte de } \\
\text { forma rutinaria. }\end{array}$ & 6 & 60.07 & 18.33 & 21.60 & $\begin{array}{l}\text { Parece que el 40\% (sumando } \\
\text { los indecisos) no tienen una } \\
\text { buena razón (o no saben). }\end{array}$ \\
\hline $\begin{array}{l}\text { El reporte hace que se } \\
\text { exponga mi práctica clínica de } \\
\text { manera innecesaria. }\end{array}$ & 7 & 65.77 & 15.19 & 19.04 & $\begin{array}{l}\text { Casi dos tercios están } \\
\text { en desacuerdo con que } \\
\text { reportar pone en peligro la } \\
\text { reputación del médico (por } \\
\text { consecuencias legales). }\end{array}$ \\
\hline $\begin{array}{l}\text { Realmente no sé cómo realizar } \\
\text { un reporte de reacciones } \\
\text { adversas espontáneas. }\end{array}$ & 8 & 14.69 & 74.43 & 10.88 & $\begin{array}{l}\text { Tres cuartas partes de los } \\
\text { encuestados no saben } \\
\text { reportar. Parece que sólo el } \\
\text { 15\% sabe. }\end{array}$ \\
\hline
\end{tabular}

Tabla 3. Factores (favorables y adversos) evaluados en el sondeo: razones para no reportar.

\begin{tabular}{|c|c|c|c|c|c|}
\hline $\begin{array}{l}\text { Factor para reportar / no } \\
\text { reportar }\end{array}$ & Pregunta & $\begin{array}{l}\text { Favorable } \\
\text { (\%)en } \\
\text { desacuerdo }\end{array}$ & $\begin{array}{l}\text { Adverso }(\%) \\
\text { de acuerdo }\end{array}$ & $\begin{array}{l}\text { Indeciso/ } \\
\text { no sabe } \\
(\%)\end{array}$ & Comentarios \\
\hline $\begin{array}{l}\text { Solamente aquellas reacciones } \\
\text { adversas medicamentosas } \\
\text { que demuestran una relación } \\
\text { causa-efecto deben ser } \\
\text { reportadas. }\end{array}$ & 9 & 45.74 & 38.76 & 15.50 & $\begin{array}{l}\text { Cerca de la mitad creen que } \\
\text { es necesario que exista una } \\
\text { relación causal (incluyendo los } \\
\text { indecisos). }\end{array}$ \\
\hline $\begin{array}{l}\text { Las reacciones adversas } \\
\text { de un medicamento ya } \\
\text { son conocidas cuando } \\
\text { el medicamento llega } \\
\text { al mercado, puesto que } \\
\text { solamente se comercializan } \\
\text { medicamentos seguros. }\end{array}$ & 10 & 52.03 & 32.30 & 15.67 & $\begin{array}{l}\text { Casi la mitad piensan que las } \\
\text { RAM ya se conocen cuando un } \\
\text { medicamento entra al mercado } \\
\text { (sumando "de acuerdo" con } \\
\text { "no sabe"). }\end{array}$ \\
\hline $\begin{array}{l}\text { Los médicos debiéramos } \\
\text { contribuir al avance } \\
\text { general del conocimiento } \\
\text { médico a través del reporte } \\
\text { de reacciones adversas } \\
\text { medicamentosas. }\end{array}$ & 11 & $\begin{array}{l}87.31 \\
\text { (Favorable- } \\
\text { de } \\
\text { acuerdo) }\end{array}$ & $\begin{array}{l}4.23 \\
\text { (Adverso- en } \\
\text { desacuerdo) }\end{array}$ & 8.46 & $\begin{array}{l}\text { Casi todos los encuestados } \\
\text { están de acuerdo en que } \\
\text { deberían contribuir al } \\
\text { conocimiento médico } \\
\text { mediante los reportes. }\end{array}$ \\
\hline $\begin{array}{l}\text { En realidad es muy } \\
\text { difícil el determinar si } \\
\text { un medicamento es el } \\
\text { responsable de una reacción } \\
\text { adversa. }\end{array}$ & 12 & $\begin{array}{l}34.42 \\
\text { (Favorable- } \\
\text { de } \\
\text { acuerdo) }\end{array}$ & $\begin{array}{l}39.23 \\
\text { (Adverso- en } \\
\text { desacuerdo) }\end{array}$ & 26.35 & $\begin{array}{l}\text { Aproximadamente } 1 / 3 \text { no } \\
\text { concuerda en que sea difícil } \\
\text { determinar si un medicamento } \\
\text { es responsable de una RAM. } \\
\text { Otro tercio no lo saben. El } \\
\text { desconocimiento representa } \\
\text { casi dos tercios de las } \\
\text { respuestas. }\end{array}$ \\
\hline
\end{tabular}


Responsabilidad profesional aplicada a la farmacovigilancia: un estudio de caso en México - Germán Novoa-Heckel et al.

\begin{tabular}{|l|l|l|l|l|l|}
\hline $\begin{array}{l}\text { El reporte de reacciones } \\
\text { adversas medicamentosas } \\
\text { requiere de mucho tiempo y } \\
\text { atención. }\end{array}$ & 13 & 24.81 & 40.58 & 34.62 & $\begin{array}{l}\text { El 40\% está de acuerdo en } \\
\text { que reportar demanda mucho } \\
\text { tiempo y atención; 34\% no } \\
\text { saben si es así. }\end{array}$ \\
\hline $\begin{array}{l}\text { No sé dónde encontrar } \\
\text { el formato de Reporte } \\
\text { de Reacciones Adversas a } \\
\text { Medicamentos. }\end{array}$ & 14 & 16.38 & 73.99 & 9.63 & $\begin{array}{l}\text { Casi 84\% de los encuestados } \\
\text { no saben dónde encontrar el } \\
\text { formato. Sólo un 16\% dicen } \\
\text { saberlo. }\end{array}$ \\
\hline $\begin{array}{l}\text { El reporte es burocrático y } \\
\text { complicado. }\end{array}$ & 15 & 20.12 & 37.14 & 42.75 & $\begin{array}{l}\text { Cerca de un tercio piensan que } \\
\text { es burocrático, y 42\% no saben } \\
\text { si lo es. }\end{array}$ \\
\hline $\begin{array}{l}\text { Al reporte lo considero } \\
\text { valioso en algunos casos de } \\
\text { controversia legal. }\end{array}$ & 16 & $\begin{array}{l}73.44 \\
\text { (Favorable- } \\
\text { de } \\
\text { acuerdo) }\end{array}$ & $\begin{array}{l}\text { (Adverso-en } \\
\text { desacuerdo) }\end{array}$ & $\begin{array}{l}\text { Casi tres cuartas partes de los } \\
\text { encuestados están de acuerdo } \\
\text { en que el reporte podría ser } \\
\text { útil en casos de controversia } \\
\text { legal. }\end{array}$ \\
\hline
\end{tabular}

Tabla 4. Factores (favorables y adversos) evaluados en el sondeo: posibles soluciones y razones para reportar.

\begin{tabular}{|c|c|c|c|c|c|}
\hline $\begin{array}{l}\text { Factor para reportar / no } \\
\text { reportar }\end{array}$ & Pregunta & $\begin{array}{l}\text { Favorable- } \\
\text { en } \\
\text { desacuerdo } \\
(\%)\end{array}$ & $\begin{array}{l}\text { Adverso - } \\
\text { de acuerdo } \\
(\%)\end{array}$ & $\begin{array}{l}\text { Indeciso/ no } \\
\text { sabe } \\
(\%)\end{array}$ & Comentarios \\
\hline $\begin{array}{l}\text { El formato de reporte } \\
\text { debiera ser más amigable en } \\
\text { su diseño. }\end{array}$ & 17 & 5.30 & 57.20 & 37.50 & $\begin{array}{l}\text { Casi dos tercios quisieran } \\
\text { un formato más amigable; } \\
\text { el resto está en desacuerdo } \\
(5 \%) \text { o indeciso } \\
\text { (aproximadamente } 1 / 3 \text { ). }\end{array}$ \\
\hline $\begin{array}{l}\text { Solamente un sistema de } \\
\text { castigo-recompensa hará } \\
\text { que los médicos reporten } \\
\text { las reacciones adversas a los } \\
\text { medicamentos. }\end{array}$ & 18 & 52.30 & 26.89 & 20.81 & $\begin{array}{l}\text { La mitad está en desacuerdo; } \\
\text { más del } 20 \% \text { no sabe. }\end{array}$ \\
\hline $\begin{array}{l}\text { Con el reporte se puede } \\
\text { contribuir al conocimiento } \\
\text { médico. }\end{array}$ & 19 & $\begin{array}{l}88.75 \\
\text { (Favorable- } \\
\text { de acuerdo) }\end{array}$ & $\begin{array}{l}3.32 \\
\text { (Adverso-en } \\
\text { desacuerdo) }\end{array}$ & 7.93 & $\begin{array}{l}\text { Confirmación de la pregunta } \\
\# 14 \text {. El reporte puede } \\
\text { contribuir al conocimiento } \\
\text { médico. }\end{array}$ \\
\hline $\begin{array}{l}\text { Solamente cuando hay una } \\
\text { relación evidente entre el } \\
\text { medicamento y la reacción } \\
\text { se debe reportar. }\end{array}$ & 20 & 33.77 & 50.93 & 15.30 & $\begin{array}{l}\text { Más de la mitad creen } \\
\text { (erróneamente) que debe } \\
\text { haber una relación entre la } \\
\text { reacción y el fármaco para } \\
\text { emitir un reporte. Cerca del } \\
15 \% \text { están indecisos. }\end{array}$ \\
\hline $\begin{array}{l}\text { Es la severidad de la reacción } \\
\text { la que decide si debemos } \\
\text { o no reportar la reacción } \\
\text { adversa medicamentosa. }\end{array}$ & 21 & 54.87 & 26.97 & 18.16 & $\begin{array}{l}\text { Más de la mitad reconocen } \\
\text { que no es necesario que la } \\
\text { reacción sea severa para que } \\
\text { se reporte. }\end{array}$ \\
\hline $\begin{array}{l}\text { Solamente deben reportarse } \\
\text { reacciones adversas } \\
\text { previamente desconocidas. }\end{array}$ & 22 & 60.07 & 25.37 & 14.55 & $\begin{array}{l}\text { Más del } 60 \% \text { sabe que } \\
\text { las reacciones adversas } \\
\text { previamente desconocidas } \\
\text { deben reportarse. }\end{array}$ \\
\hline $\begin{array}{l}\text { Tiene que educarse lo } \\
\text { relacionado con el reporte } \\
\text { en la facultad para que } \\
\text { funcione. }\end{array}$ & 23 & 11.57 & 74.00 & 14.42 & $\begin{array}{l}\text { Más de } 2 / 3 \text { piensan que } \\
\text { sólo a través de la educación } \\
\text { temprana se puede resolver } \\
\text { el problema. (desfavorable } \\
\text { para el sistema) }\end{array}$ \\
\hline
\end{tabular}




\begin{tabular}{|l|l|l|l|l|l|}
\hline $\begin{array}{l}\text { Realmente no sé cuál es el } \\
\text { alcance del reporte ante las } \\
\text { autoridades. }\end{array}$ & 24 & 13.22 & 70.20 & 16.57 & $\begin{array}{l}\text { Casi el 90\% desconoce los } \\
\text { alcances de los reportes. }\end{array}$ \\
\hline
\end{tabular}

Tabla 5. Factores que intervienen en la falta de reportes (conocimiento y actitudes, favorables y adversos) según lo indagado en el cuestionario.

\begin{tabular}{|c|c|c|c|c|c|c|}
\hline Factor & Pregunta & $\begin{array}{l}\text { Favorable } \\
(\%)\end{array}$ & $\begin{array}{l}\text { Adverso } \\
(\%)\end{array}$ & $\begin{array}{l}\text { Indeciso } \\
(\%)\end{array}$ & $\begin{array}{l}\text { Ponderación } \\
\text { relativa } \\
(\%) \text { debida } \\
\text { a distintos } \\
\text { factores } \\
\text { (consenso, } \\
\text { ref. }[12] \text { ) }\end{array}$ & Comentarios \\
\hline Ignorancia & $8,9,21,22$ & 43.72 & 41.72 & 25.29 & 95 & $\begin{array}{l}\text { Se observa ignorancia en } \\
\text { más de dos tercios de los } \\
\text { participantes, si se incluye a } \\
\text { los indecisos. }\end{array}$ \\
\hline Autosuficiencia & 10,20 & 43.89 & 42.02 & 14.08 & 47 & $\begin{array}{l}\text { Aproximadamente la } \\
\text { mitad de los encuestados } \\
\text { demuestra autosuficiencia/ } \\
\text { ignorancia. }\end{array}$ \\
\hline Miedo & 7,16 & 69.36 & 15.02 & 17.13 & 24 & $\begin{array}{l}\text { No se encuentra miedo en } \\
\text { más de dos tercios de los } \\
\text { encuestados. }\end{array}$ \\
\hline Indiferencia & 11,19 & 88.38 & 3.75 & 7.86 & 67 & $\begin{array}{l}\text { No se halla indiferencia } \\
\text { en el } 88.38 \% \text { de los } \\
\text { participantes. }\end{array}$ \\
\hline Inseguridad & 12 & 32.63 & 40.61 & 26.76 & 67 & $\begin{array}{l}\text { Más de la mitad de los } \\
\text { encuestados no muestran } \\
\text { inseguridad (incluyendo a } \\
\text { los indecisos). }\end{array}$ \\
\hline Apatía & $\begin{array}{l}4,6,13,14 \\
15,17\end{array}$ & 38.38 & 32.89 & 28.72 & 77 & $\begin{array}{l}\text { Se encuentra apatía en } \\
\text { más de dos tercios de los } \\
\text { encuestados cuando se } \\
\text { incluye a los indecisos. }\end{array}$ \\
\hline
\end{tabular}

(Nota: no se indagó sobre culpa, ambición de publicar, incentivos económicos y timidez, ya que estos factores no se consideraron relevantes en México al momento del estudio.) 
Responsabilidad profesional aplicada a la farmacovigilancia: un estudio de caso en México - Germán Novoa-Heckel et al.

\section{Referencias}

1. Jonas H. El Principio de Responsabilidad. Ensayo de una ética para la civilización tecnológica. Barcelona: Editorial Herder; 1995: 23.

2. Rodríguez-Betancourt JL, García-Vigil JL, Giral-Barnés C, Hernández-Santillán D, Jasso-Gutiérrez L: Farmacovigilancia I. El inicio. Rev Med IMSS 2004, 42: 327-329.

3. WHO Collaborating Centre for International Drug Monitoring. Viewpoint Part 1. Uppsala, Sweden: The Uppsala Monitoring Centre; 2002.

4. México. NOM-220-SSA1-2002, Instalación y Operación de la Farmacovigilancia. México, D.F.: Diario Oficial de la Federación; 2004.

5. Parkin DM, Clayton D, Black RJ, et al. Childhood leukaemia after Chernobyl: 5 year follow-up. British Journal of Cancer 1996; 73: 1006-1012.

6. Lopez-Gonzalez E, Herdeiro MT, Figueiras A: Determinants of under-reporting of adverse drug reactions: a systematic review. Drug Saf 2009; 32: 19-31.

7. Inman WH: Attitudes to adverse drug reaction reporting. Br J Clin Pharmacol. 1996, 41(5):434-435.

8. Inman WH: Assessment of drug safety problems. In Gent M, Shigmatsu I, (eds.) Epidemiological Issues in Reported Drug-Induced Illnesses. Honolulu: McMaster University Library Press; 1976: 17-24.

9. Inman WH, Weber JCT. The United Kingdom. In Inman WH, (ed.) Monitoring for Drug Safety. Lancaster: MTP Press Ltd; 1986: 13-47.

10. Golomb BA, McGraw JJ, Evans MA, Dimsdale JE: Physician response to patient reports of adverse drug effects: implications for patient-targeted adverse effect surveillance. Drug Saf 2007; 30: 669-675.

11. Herdeiro MT, Polonia J, Gestal-Otero JJ, Figueiras A. Factors that influence spontaneous reporting of adverse drug reactions: a model centralized in the medical professional. J Eval Clin Pract 2004, 10: 483-489.

12. Blenkinsopp A, Wilkie P, Wang M, Routledge PA. Patient reporting of suspected adverse drug reactions: a review of published literature and international experience. Br J Clin Pharmacol 2006; 63: 148-156.

13. Marshall G. The purpose, design and administration of a questionnaire for data collection. Radiography 2005; 11: 131136.

14. Spector PE. Summated Rating Scale Construction: An Introduction. Newbury Park, CA: Sage Publications; 1992.

15. DeVellis R. Scale Development: Theory and Applications. Thousand Oaks, CA: Sage Publications; 2003.

16. Carmines EG, Zeller RA. Reliability and Validity Assessment. Newbury Park, CA: Sage Publications; 1979.

17. Softonic. SPSS para Windows. Disponible en http://www.softonic.com/s/spss

18. Cronbach LJ. Test validation. In Thorndike RL, (ed.) Educational Measurement. Washington, DC: American Council on Education; 1971: 443-507.

19. Cronbach LJ. Coefficient alpha and the internal structure of tests. Psychometrika 1951; 16: 297-334.

20. U.S. Food and Drug Administration. Center for Education and Research on Therapeutics. Preventable Adverse Drug Reactions: A Focus on Drug Interactions. Available from http://www.fda.gov/drugs/developmentapprovalprocess/developmentresources/druginteractionslabeling/ucm110632.htm . Accessed on: April 15, 2013.

21. Islam AF. Improving drug utilization: what about the KAP gap? Tropical Doctor 1993; 23: 89-90

22. Hong YP, Kwon DW, Kim SJ, et al: Survey of knowledge, attitudes and practices for tuberculosis among general practitioners. Tuberculosis and Lung Disease 1995; 76:431-435.

23. Blenkinsopp A, et al. Patient reporting of suspected adverse drug reactions: a review of published literature and international experience. Br. J. Clin. Pharmacol 2007; 63: 148-156.

24. Aagaard L, Strandell J, Melskens L, Petersen PS, Holme Hansen E. Global patterns of adverse drug reactions over a decade: analyses of spontaneous reports to VigiBase ${ }^{\mathrm{TM}}$. Drug Saf 2012; 35(12): 1171-1182.

Recibido: 4 de septiembre de 2014

Aceptado: 10 de noviembre de 2014 